\title{
Errores más comunes al redactar artículos médicos originale
}

\author{
Adriana Hernández-Vargas, ${ }^{1}$ Francia Estefanía Pérez-Manjarrez, ${ }^{1}$ Indira Rocío Mendiola-Pastrana, ${ }^{2}$ \\ Eduardo López-Ortiz y Geovani López-Ortiz* \\ ${ }^{1}$ Universidad Nacional Autónoma de México, Facultad de Medina, Subdivisión de Medicina Familiar; ${ }^{2}$ Instituto Mexicano del Seguro Social, Uñidad
} de Medicina Familiar 28. Ciudad de México, México

\section{Resumen}

Una de las habilidades que más requiere ser fortalecida en el área médica es la difusión de conocimiento, por tal motivo, es necesario brindar elementos que promuevan la capacitación en redacción científica. Identificar cuáles son los problemașmás comunes al redactar artículos médicos originales para su publicación proporcionará recursos útiles que contribuyan a lấgeneración de conocimiento. Existen diversos motivos que intervienen para que un artículo original no sea aceptado en revista médica, entre los que destacan falta de apego a las instrucciones para autores, problemas metodológicos y de diseño, así como de estructura, sin embargo, el aspecto que más influye y pocas veces se señala, es la redacción inadecuada de los escritos de investigación, la cual se hace patente en las diferentes secciones que componen los manuscritos. Todoš los factores mencionados son responsabilidad de los autores, por lo que deben ser atendidos apropiadamente. El presentesescrito tiene como objetivo señalar los errores más comunes al redactar escritos médicos originales, los cuales, de ser evitälos, incrementarán la posibilidad de que sus trabajos de investigación sean aceptados para su publicación.

PALABRAS CLAVE: Publicación. Manuscritos. Rechazo. Revisión por pares. Artículo de revista.

\section{Abstract}

One of the skills that is more in need to be strengthened in the medical area is the dissemination of knowledge. For this reason, it is necessary to provide elements that promote training in scientific writing. Identifying the most common problems when writing medical original articles for publication will provide useful resources that should contribute to the generation of knowledge. There are multiple reasons that intervene for an original article not to be accepted in a medical journal, among which failure to follow instructions for authors and methodological, design and structural problems stand out. However, the aspect that most influences and that is seldom pointed out, is inadequate writing of research manuscripts, which becomes evident in the different sections that make up manuscripts. All the above-mentioned factors are the responsibility of the authors, aand therefore must be taken care of properly. The purpose of this article is to identify the most common mistakes when winting original medical manuscripts, which, if avoided, will increase the possibility for research papers to be accepted for publication.

KEY WORDS: Publication. Manuscripts. Rejection. Peer review. Journal article.

Fecha de recepción: 19-03-2019

Fecha de aceptación: 22-03-2019

DOI: 10.24875/GMM.19005172
Gac Med Mex. 2019;155:635-640 Disponible en PübMed www.gacetamedicademexico.com 
La publicación científica es el medio de comunicación más amplio para la difusión de hallazgos en la comunidad médica, la meta de todo proyecto de investigación es, o debería ser, que sus resultados puedan difundirse de forma exitosa,,$^{1,2}$ sin embargo, una parte importante de escritos médicos no llega a publicarse después de someterse a revistas especializadas. El índice de rechazo en algunas revistas llega hasta $96 \%,{ }^{3,4}$ atribuido, en parte, a los errores en la estructura de los escritos. ${ }^{5}$

Antes de someter un artículo para su publicación se debe considerar que un protocolo de estudio basado en un pregunta de investigación irrelevante, con diseños metodológicos pobres, ausencia de información, valores muestrales pequeños, uso inadecuado de pruebas estadísticas, falta de actualización y alcance, conflictos de interés, puede ser desacreditado por el editor desde un inicio., ${ }^{6,7}$ En una decisión negativa también influye la falta de apego al modelo estándar de artículos originales (Introducción, Métodos, Resultados y Discusión), así como los criterios editoriales. ${ }^{8-11}$ No obstante, el motivo principal de rechazo está asociado con una redacción deficiente, la cual, de acuerdo con editores y revisores, impacta preponderantemente en la calidad y presentación de los manuscritos, independientemente del contenido o novedad de los hallazgos reportados en el estudio. ${ }^{9-13}$

La mayoría de los autores escribe por aprendizaje individualizado y mediante ensayo y error, esto se debe a que en las universidades no existen cursos formales de redacción o presentación de trabajos científicos para su publicación; ${ }^{14}$ pese a estas limitaciones, la responsabilidad de escribir adecuadamente recae directamente en los autores. Los hallazgos de una investigación siempre deben ser comunicados en forma efectiva y clara. ${ }^{10,13,15-17}$

Es fundamental identificar el tema central del escrito, para precisar el tipo de audiencia y la revista adecuada para su envío. Muchos autores tienden a someter sus artículos a revistas a las que no corresponde el alcance de su trabajo, es decir, la investigación realizada no es compatible con la temática o el impacto de la revista. Una vez identificada la revista, los autores deben leer y apegarse a las normas editoriales, aspecto que, a pesar de resultar obvio, no siempre es considerado. ${ }^{18,19}$

Debido a la necesidad de fomentar y fortalecer las habilidades de redacción científica en médicos especialistas y en formación, el objetivo de este escrito es señalar los errores más comunes al redactar manuscritos originales para ser publicados en revistas médicas especializadas. A continuación, se señalan dichos errores de acuerdo con las diferentes seçciones que componen los artículos de investigación?

\section{Título}

Redactar un título adecuado es de gran trascendencia debido a que es la primera impresión que el editor y revisores tendrán del artículo. Su importancia radica en que debe generar inmediatamente interés en lectores e identificar el contenido del manuscrito se redacta en presente simple. Los errores más frecuentes en su elaboración son: ${ }^{1,2,10,15,20-23}$

- Títulos demasiado largos (> 15 palabras) cortos.

- Título poco atractivo al lector.

- El título no concuerda con el tema y el objetivo del estudio.

- Título incomprensible, redundante o incompleto.

- Título con faltas de ortografía.

- Uso de abreviaturas o siglas.

- Uso indebido de mayúsculas.

\section{Resumen}

En el resumen se encuentran los elementos clave de la investigación, es el segundo elemento más čnsultado. Su correcta elaboración atraerá al lector para concluir la lectura total del escrito; se realiza al final del artículo para transmitir correctamente la esencia de este. Es redactado en el tiempo verbal correspondiente para cada sección. Los problemas más cợnunes en su redacción son:2,9,10,16,17,21,22

- Errores de extensión: con frecuencia es de 200 o 250 palabras o muy corto.

- Sus apartados no se ajustan a los señaladosīpor las revistas.

- No sintetiza apropiadamente los aspectos.más relevantes del estudio.

- Se copia y pega fragmentos de las distintas secciones del escrito.

- Contiene información que no se menciona en el resto del artículo.

- Redacción a manera de introducción o conclusión.

- Se incluyen abreviaturas sin significado.

- Se incluyen citas en este apartado.

\section{Palabras clave}

Las palabras clave son una herramienta importānte que optima la búsqueda rápida de resultados por 
temas en diferentes bases de datos electrónicas como PubMed, Web of Science o Google Scholar, entre otras. ${ }^{6,15}$ Los errores más comunes son: ${ }^{1,2,6,23,24}$

- Omisión de palabras clave.

- No se utilizing términos MeSH (Medical Subject Headings).

- Número insuficiente de palabras clave (dependiendo de la revista se utilizan de 3 a 8).

- Se utilizan las mismas palabras que se ocuparon en el título.

- No se relacionan con el tema de estudio.

- Uso inadecuado de palabras compuestas.

\section{Introducción}

La introducción delimita la importancia y pertinencia del estudio y establece su justificación mediante un análisis bibliográfico crítico y actual, en ella se determina el objetivo de la investigación. ${ }^{2,18}$ Debe ser redactada en presente para hallazgos actualmente aceptados y en pasado para describir métodos previamente utilizados en otros artículos y declaraciones que ya no son consideradas verdaderas. ${ }^{21}$ Los errores más frecuentes en su elaboración son:2,15,17,18

- Presenta problemas de extensión, supera $15 \%$ del artículo.

- No define claramente la importancia de la investigación, ni el contexto de esta en el ámbito internacional y local.

- No cita las fuentes empleadas en el desarrollo de la introducción.

- Redacción informal, subjetiva, con problemas gramaticales y desvinculados del título y objetivo.

- Uso de citas innecesarias, insuficientes o inadecuadas para el tema de estudio.

- Utiliza abreviaturas no oficiales, improvisadas o no las definen.

- No señala el objetivo de la investigación.

\section{Métodos}

La sección de método representa el fundamento experimental del manuscrito, ${ }^{1}$ describe el proceso mediante el cual se realizó la investigación, proporciona información y detalles específicos para que el estudio sea reproducido bajo las mismas condiciones de trabajo. ${ }^{18}$ Su redacción siempre es en pasado simple..$^{21,22}$ Con frecuencia se observan errores en su elaboración originados desde la conceptualización del diseño de estudio, dichos errores son: ${ }^{2,9,10,15,16,20,23}$
- No se señala el tipo de estudio.

- Elección incorrecta del tipo de estudio pararesponder la pregunta de investigación.

- Se omite el periodo, lugar y fechas del estudio.

- Falta de descripción del tamaño de muestra, i̦ipo de muestreo y variables.

- Omite los criterios de selección (inclusión, exelusión y eliminación).

- No se señala la aprobación por el comité dèética, ni la firma de consentimiento informado.

- Se utilizan instrumentos no validados para läpoblación de estudio.

- No se describe adecuadamente qué variables miden los instrumentos aplicados, ni sus puñtos de corte.

- No se señala adecuadamente el uso ni la pêdrinencia de las pruebas estadísticas utilizadas, ni su significancia.

- No se mencionan las guías o manuales utilizados para referir parámetros clínicos.

- Se indica instrumentos, clasificaciones o parámetros clínicos obsoletos.

- Falta de controles u otras medidas de sesgo.

\section{Resultados}

En la sección de resultados se resaltan conceétamente los hallazgos del estudio, siempre tomändo como guía el objetivo del trabajo, no debe contener interpretaciones o cometarios personales. Los artícullos con deficiencias en los resultados generan múltiples inconvenientes, los cuales disminuyen el impacto- $\mathrm{del}$ trabajo de investigación. ${ }^{25}$ Es adecuado que los résultados sean redactados en pasado, en forma excepcional se puede utilizar presente. ${ }^{21,26}$ Los errores más Comunes encontrados en este apartado son:10,15,16

- Los resultados no están relacionados con elöobjetivo del trabajo.

- Se describen procedimientos y pruebas estadísticas no mencionados en los métodos.

- Se utilizan pruebas estadísticas incorrectamente.

- Las tablas, gráficas o figuras no están justificadas en el texto.

- Repite la misma información en el texto, tablăg y figuras.

- Los números absolutos reportados no acompañan de porcentajes.

- Hay datos perdidos y valores en tablas que al sumar no corresponden al resultado total.

- Hay discontinuidad de resultados respectó a cómo fueron presentados en los métodos. 
- Se presentan datos o parámetros que carecen de unidades de medida.

- Se incorporan citas.

\section{Discusión}

Dependiendo de la revista, la conclusión y la discusión pueden están contenidas en el mismo apartado, mientras que en algunas ocasiones se exige una clara división entre ambas. ${ }^{6}$ La discusión contextualiza los hallazgos más relevantes de la investigación en relación con la literatura actual internacional, en ella, el autor expresa objetivamente la interpretación de los resultados, para dar respuesta a la pregunta de investigación. ${ }^{9,10}$ Su redacción debe ser en pasado para referirse a métodos 0 resultados y en presente para expresar el análisis e interpretación de los resultados obtenidos. ${ }^{21,26} \mathrm{~A}$ continuación se mencionan los errores más frecuentes:9,10,16,23

- No se explica cómo los resultados obtenidos contribuyen al fortalecimiento de la disciplina médica, ni mencionan las ventajas de su aplicabilidad.

- No se discuten puntualmente las tablas, gráficos o figuras.

- Se sobrevaloran los resultados.

- Solo se describen extensamente los resultados de otras investigaciones.

- Se comparan trabajos con poca relación con la temática abordada.

- Se señalan hallazgos estadísticos sin un soporte significativo que los avale.

- Se señalan hallazgos no abordados en métodos y resultados.

- Se resta importancia a los hallazgos más significativos o se interpretan en forma incorrecta.

- Se incluyen opiniones subjetivas o parciales (sesgo).

- Se emplean citas insuficientes y desactualizadas (se recomienda sean menores a cinco años).

- No se mencionan los alcances y limitaciones del estudio.

\section{Conclusión}

La conclusión debe contener los hallazgos más relevantes de la investigación y su contribución al conocimiento actual, propone futuras líneas de investigación y está centrada en el objetivo del estudio. ${ }^{6,16,23}$ Es correcto redactar en tiempo presente los hallazgos más relevantes y en futuro al sugerir nuevas investigaciones..$^{21,26}$ Los errores más comunes en este apartado son: $2,6,15,16,23$
- Conclusiones demasiado extensas o muy breves.

- Se realizan generalidades de aspectos relacionados con el trabajo.

- Las reflexiones no se centran en los objetivỗs y resultados del estudio.

- Se realizan juicios subjetivos sobre el aporte de la investigación.

- No se enfatizan los hallazgos más relevantes del estudio.

- Se realiza un resumen de todo el artículo.

- No se presenta una propuesta breve que atenúe la problemática estudiada.

\section{Referencias}

El objetivo de las referencias es proveer una Iista de fuentes de información actuales e internacionales relacionadas con el manuscrito y que proporcionan sustento científico al contenido presentado. ${ }^{6}$ La mayor cantidad de errores se detectan en esta sección, debido a que los autores no ponen énfasis en su ellaboración. ${ }^{23}$ La problemática más frecuente en südesarrollo es la siguiente: $2,9,10,12,15,23,27$

- Se citan en un formato distinto al señalado la revista o utilizan diversos estilos de citaciön.

- Se utiliza un número insuficiente o excesivō de citas para todo un artículo.

- Se citan trabajos no relacionados con el tema de estudio.

- Se utilizan referencias desactualizadas (> 5 años).

- Se citan resúmenes o trabajos sin sustento científico.

- Falta de numeración, presentación desordenada y ausencia de correlación de las citas en el téxto y en las referencias.

- Se indican referencias inexistentes.

- Las citas están incompletas.

\section{Recursos gráficos}

Las tablas, imágenes, cuadros, gráficas y algōitmos incluidos en los artículos son elementos ilustrativos que facilitan la lectura y comprensión del escrî̉o.16 Son útiles para organizar, sintetizar, comparar yeesquematizar los resultados. ${ }^{27}$ Los errores más comunes en estos recursos son: $2,16,20,27$

- Referencia a tablas, cuadros o figuras inexistentes.

- Uso excesivo o deficiente de recursos gráficos (la revista puede señalar la cantidad máximā de estos). 
- Omisión de títulos y notas explicativas que definen los recursos gráficos.

- No conservan una secuencia numérica correcta.

- Envío de imágenes y tablas en formatos electrónicos inadecuados o con una resolución deficiente (los parámetros estándar son de 300 puntos por pixel o dpi y dependiendo la figura o imagen las medidas mínimas son de $12 \mathrm{~cm}$ ).

- Utilizan fotografías de pacientes sin su consentimiento informado.

- Uso de recursos gráficos que no fueron explicados ni justificados en el escrito.

- No se señala a qué corresponden los ejes " $X$ " y "Y" o en estos ejes se refieren valores incorrectos.

\section{Consideraciones generales}

Es necesario mencionar que existen conductas inadecuadas relacionadas con la ética de los autores; como el copiado y pegado de información y el plagio. En los últimos años se ha observado mayor cantidad de manuscritos en los cuales se ha detectado plagio a diferentes niveles. ${ }^{28,29}$ El plagio representa una grave conducta ética y académica en la investigación y es más frecuente de lo que se ha dimensionado. ${ }^{29-32}$ Se define como el acto de utilizar ideas o palabras publicadas o no publicadas de personas o grupos de trabajo, así como el uso de otra propiedad intelectual y presentarlas como originales. ${ }^{29,33-35}$ Puede ir desde frases o párrafos cortos hasta textos completos. ${ }^{30}$ Su mención es importante ya que representa también una causa de rechazo de artículos.

Al momento de someter un artículo para su publicación se debe evitar en todo momento lo siguiente: ${ }^{9,10,12,29,36-40}$

- Plagio o autoplagio.

- Reenviar artículos previamente rechazados a la misma revista.

- Enviar simultáneamente el mismo artículo a varias revistas.

- Falta de información sobre adscripciones, autor de correspondencia, nombres completos y contribución de los autores, no conflicto de interés o apoyos otorgados.

- Desconocer todos los documentos que solicitan las revistas para que el artículo quede registrado debidamente, como carta de presentación, registros de los comités de ética e investigación, carta de no conflicto de intereses o carta de cesión de derechos, entre otros.

\section{Conclusiones}

Debido a la escasa formación académica en redacción científica en los diferentes planes de estudiō de las escuelas de medicina y en el ámbito médico es necesario fortalecer y sistematizar los aspectos clave en la generación y difusión de conocimiento; la@elaboración de escritos médicos es una actividad gratificante, sin embargo, también es ardua y compleja, por lo que requiere un alto de compromiso por părte de todos los autores. Los errores de redacción presentes en las diferentes secciones que componeñlos escritos médicos originales representan una causa frecuente de rechazo, por lo que requieren ser atendidos y evitados antes de someter un artículo a tuna revista especializada.

\section{Bibliografía}

1. Downey SM, Geraci SA. Manuscript development and publishing: a 5-step approach. Am J Med Sci. 2017;353:132-36.

2. Venkatesan S. Common errors in scientific paper submissions: $\mathrm{a}$ reviewer's report. J Soc Sci. 2014;41:279-293.

3. For authors [En línea]. EE. UU: JAMA Network; 2019.

4. Redberg RF. JAMA Internal Medicine: the year in review, 2017. JAMA Intern Med. 2018;178:456-457.

5. Hobson AJ. Why manuscripts submitted to an international peer reviewed journal in education are rejected. IJMCE. 2014;3:188-196.

6. Maiorana FA, Mayer HF. How to avoid common errors in writing scientific manuscripts. Eur J Plast Surg. 2018;41:489-494.

7. Pimm J. Dear editor, why have you rejected my article? The Psychiatrist. 2013;37:313-314.

8. Pierson DJ. The top 10 reasons why manuscripts are not accepted for publication. Respir Care. 2004:49:1246-1252.

9. Ezeala CC, Nweke IN, Ezeala MO. Common errors in manuscriptssubmitted to medical science journals. Ann Med Health Sci Res, 2013;3: 376-379.

10. Khatri N, Varma A, Budhwar P. Commonly observed shortcomings in manuscripts submitted to management journals. IIMB ManagementRev. 2017;29:203-209.

11. Pinto N. Common errors to look out for in medical papers. Euro SGitedit. 2013:39:69.

12. Phani VK, Rao CS. A review of reasons for rejection of manuscripts. JRSP-ELT. 2018:8:1-9.

13. Mack CA. How to write a good scientific paper: a reviewer's checklist. J Micro/Nanolithography MEMS MOEMS. 2015;14:020101.

14. Grindstaff TL, Saliba SA. Avoiding manuscript mistakes. Int J Šports Phys Ther. 2012;7:518-524.

15. Ohwovoriole AE. Writing biomedical manuscripts. Part II: Standarefelements and common errors. West Afr J Med. 2011;30:389-399. .

16. Understanding the publishing process: how to publish in scholarlyournals [En línea]. Países Bajos: Elsevier; 2019.

17. Grech V. WASP (write a scientific paper): miscellaneous practicab and material aspects. Early Hum Dev. 2019;128:105-106.

18. Iskander JK, Wolicki SB, Leeb RT, Siegel PZ. successful scientific writing and publishing: a step-by-step approach. Prev Chronic Dis. 2018; 15:1-6.

19. Sullivan GM. What to do when your paper is rejected. J Grad MediEduc. 2015;7:1-3.

20. Adeosun OO, Kayode MO. An appraisal on common errors/mistakes in scientific writing. Int J Innov Res Sci Eng Technol. 2016;5:155-15

21. Nature [sitio web]. Effective writing. EE. UU: Scitable/Nature Education; 2019.

22. Yusoff MSB. ABC of manuscript writing. Educ Med J. 2018;10:61-67.

23. Vitse CL, Poland GA. Writing a scientific paper-a brief guide for new investigators. Vaccine. 2017;35:722-728.

24. National Library of Medicine. Medical subject headings [En líneałt. EE. UU.: National Library of Medicine; 2018.

25. Porcino A, Moraska A. Avoiding common writing mistakes that make-your editors and reviewers cringe. Int J Ther Massage Bodywork. 2015:8;1-3.

26. Silvia PJ. Write it up: practical strategies for writing and publishing journal articles. EE. UU: American Psychological Association; 2015. 
27. British Journal of Cancer. Guide to authors [En línea]. Inglaterra: British Journal of Cancer; 2018.

28. Rohwer A, Wager E, Young T, Garner P. Plagiarism in research: a survey of African medical journals. BMJ Open. 2018;8:e024777.

29. Bauchner H, Fontanarosa PB, Flanagin A, Thornton J. Scientific misconduct and medical journals. JAMA. 2018;320:1985-1987.

30. Higgins JR, Lin FC, Evans JP. Plagiarism in submitted manuscripts: incidence, characteristics and optimization of screening-case study in a major specialty medical journal. Res Integrity Peer Rev. 2016;1:13.

31. Yadav S, Rawal G, Baxi M. Plagiarism-a serious scientific misconduct. Int J Health Sci Res. 2016:6:364-366.

32. Shewan LG, Coats A, Henein M. Requirements for ethical publishing in biomedical journals. Intern Cardiovasc Forum J. 2015;1:2.

33. Vercellini $P$, Buggio L, Viganò $P$, Somigliana $E$. Peer review in medica journals: beyond quality of reports towards transparency and public scrutiny of the process. Eur J Intern Med. 2016;31:15-19.
34. WAME Publication Ethics Committee. Recommendations on publication ethics policies for medical journals [En línea]. World Association of Medical Editors; 2019.

35. Peterson TC, Kleppner SR, Botham CM. Ten simple rules for scientists: Improving your writing productivity. PLoS Comput Biol. 2018:14:e1006379.

36. Ölçücü MT, Aydın SÜ, Özgök Y. How to write and publish a scientific article. J Urol Surg. 2018;5:63-67.

37. Cook DA. Twelve tips for getting your manuscript published. Med Teach. 2016;38:41-50.

38. Compagnoni R, Grassi A, Zaffagnini S, Bait C, Samuelsson K, Menon A, et al. A practical guide to writing (and understanding) a scientific paper: clinical studies. En: Basic methods handbook for clinical orthopaedic research. Alemania: Springer; 2019

39. Coleman B. How to write a clinical paper. En: Compagnoni R, Grasssi A, Zaffagnini S, Bait C, Samuelsson K, Menon A, et al. Basic methods handbook for clinical orthopaedic research. Alemania: Springer; 2019.

40. Light R. Why and how to write a paper? Rev Clin Esp. 2015;215:40 404 . 